\title{
Surveying the unknown forests
}

\author{
It has been calculated that $14 \%$ of the world's tree species are yet to be identified. But is that a lot or a little?
}

R igorously defining the meaning of 'tree' to include all the organisms that we happily call trees and exclude all others is not easy. According to the International Union for Conservation of Nature's Global Tree Specialist Group, a tree is "a woody plant with usually a single stem growing to a height of at least two meters, or if multi-stemmed, then at least one vertical stem five centimetres in diameter at breast height". Using this definition, Botanic Gardens Conservation International (BGCI) reported last year that there were 58,497 known species of tree, of which $30 \%$ are threatened with extinction and 142 are no longer to be found in the wild ${ }^{1}$.

Despite the prevalence of 'plant blindness', trees are conspicuous organisms that are hard to overlook. Trees can also be very long lived, with lifespans covering many centuries. The oldest known tree, a Great Basin bristlecone pine (Pinus longaeva) in the White Mountains of California, is over 4,800 years old ${ }^{2}$, and another of over 5,000 years has been claimed to exist, but cannot be confirmed. Given such visibility and permanence, the conclusion of a recently published study ${ }^{3}$ that around 9,200 species of living trees are yet to be discovered seems hard to credit.

Ancient trees are still perfectly capable of producing seedlings, and mortality rates of trees do not increase substantially with their age. This makes the existence of extremely ancient trees more likely than for other types of organism. A study in this issue ${ }^{4}$ looks at the effect on population structure of this life history and concludes that around a quarter of trees will be more than three times the median age of the population and some far more. This places a particular strain on conservation efforts as re-establishing a mature and stable population of tree species could take centuries. The authors argue that protecting ancient individuals is especially important as these winners of the 'life history lottery' preserve adaptions to rare but potentially devastating environmental extremes.

Such a long-tailed age distribution leads to many tree species having small and geographically concentrated populations. The BGCI report, for example, found that 34,575 species ( $58 \%$ of all species identified) were present in only one country. Such species with low population numbers and endemic to a single location are easy to overlook, especially in poorly surveyed areas.

To counteract this problem, Gatti et al. used the known distributions of trees to predict the global occurrence of trees, both known and unknown. They employed a branch of mathematics dealing with 'inverse probability' - also known as Bayesian statistics, after Thomas Bayes, the English Presbyterian minister and mathematician who derived its foundational theorem in the eighteenth century. An archetypal problem in inverse probability involves a jar from which black and white balls are removed one by one. On the basis of the colours of the retrieved balls, the probable distribution of black and white balls in the jar can be calculated. For the tree species, however, the jar is effectively filled with an unknown number of balls in an unknown number of colours, some of which may only occur once. This type of question was successfully tackled by Alan Turing and Irving Wood during the second World War while trying to crack the German Enigma codes. Since the 1980s the Taiwanese statistician Amy Chao has been developing the approach for the use of ecologists.

Gatti et al. divided the land surface of the globe into $10,000-\mathrm{km}^{2}$ squares and populated these from several observational databases, including the Global Forest Biodiversity Initiative (https://gfbinitiative. net/) and TreeChange (https://bio.au.dk/ en/research/research-centres/biochange/ research/treechange). The combined dataset contained records of approximately 38 million trees from 28,192 species at more than 105,000 locations. The resulting global map of species richness predicts that there are around 72,000 tree species in total, of which $43 \%$ are in South America, almost twice as many as any other continent. South America is also predicted to harbour the highest number of as yet undiscovered species.

That we are yet to discover some $14 \%$ of tree species seems difficult to credit when so much of the Earth's surface has been explored. These missing species are predicted to have tiny populations occurring in highly localized areas. That these sites seem also to be most threatened by climate change and habitat destruction makes it depressingly likely that these trees may remain permanently unknown.

Before becoming too downhearted, spare a thought for the unfortunate biologists who study other divisions of life. Entomologists have catalogued over a million insect species, but that leaves four-and-a-half million still to be identified ${ }^{5}$. Or our mycologist colleagues, for whom even $20 \%$ coverage seems an unattainable goal; only 120,000 fungal species are known from a total conservatively estimated to be between 2.2 and 3.8 million $^{6}$. Against such numbers, $86 \%$ coverage of trees seems something to celebrate.

Published online: 22 February 2022 https://doi.org/10.1038/s41477-022-01106-0

References

1. BGCI. State of the World's Trees (BGCI, 2021).

2. Salzer, M. \& Baisan, C. Dendrochronology of the "Currey Tree." in Second American Dendrochronology Conference (2013); https://ameridendro.ltrr.arizona.edu/contributionDisplay.py? contribId $=59 \&$ session $I d=9 \&$ confld $=0$

3. Gatti, R. C. et al. The number of tree species on Earth. Proc. Natl Acad. Sci. USA 11, e2115329119 (2022)

4. Cannon, C. H., Piovesan, G. \& Munné-Bosch, S. Old and ancient trees are life history lottery winners and vital evolutionary resources for long-term adaptive capacity. Nat. Plants https://doi. org/10.1038/s41477-021-01088-5 (2022).

5. Stork, N. E. Annu. Rev. Entomol. 63, 31-45 (2018).

6. Hawksworth, D. L. \& Lücking, R. Microbiol. Spectr. https://doi. org/10.1128/microbiolspec.FUNK-0052-2016 (2017). 